\title{
THE FIELD DEPENDENCE OF THE DOMAIN PERIOD IN CoCr FILMS
}

\author{
J. Kaczér $\left({ }^{1}\right)$, J. Šimšová $\left({ }^{1}\right)$, R. Gemperle $\left({ }^{1}\right)$, L. Murtinová $\left({ }^{1}\right)$ and J. C. Lodder $\left({ }^{2}\right)$ \\ ${ }^{1}$ ) Institute of Physics, Czechoslovak Academy of Sciences, Na Slovance 2, CS-180 40 Praha 8, Czechoslovakia \\ $\left(^{2}\right)$ University of Twente, P.O.B. 217, NL-7500 AE Enschede, The Netherlands
}

Abstract. - The dependence of the submicron domain period of $\mathrm{CoCr}$ films in ascending and descending fields (dc: 8-320 $\mathrm{kA} / \mathrm{m}$; superposed ac: $0-55 \mathrm{kA} / \mathrm{m}$ ) normal to the surface was investigated using the colloid-SEM method. Low coercivity samples $\left(H_{\mathrm{c}} / H_{\mathrm{k}} \sim 0.02\right)$ were measured. Comparison with calculations furnished fair agreement in contradistinction to samples havig $H_{\mathrm{c}} / H_{\mathrm{k}} \sim 0.05$. The exchange constant $A$ was determined from the thickness dependence of the domain period.

CoCr films are potential media for high density perpendicular magnetic recording. In order to understand the recording mechanism in more detail it is necessary to study the magnetization processes of these materials and especially the microscopic origins responsible for this behaviour. Schmidt and Hubert [1] used the Kerr technique to observe the domains in CoCr films. To circumvent the complexities of this method we applied the dried colloid technique first published by Craik and Griffiths [2], later improved by Goto [3]. Using his colloid-scanning electron microscope (SEM) technique we earlier reported submicron domain observations in a $\mathrm{CoCr}$ film [4]. The present paper reports the investigation of the field dependence of the domain structure of low coercivity films and the thickness dependence of the domain period.

We measured the hysteresis loops of six films given in table I; four having long- (low $H_{\mathrm{c}}$ ) and two short stripes (medium $H_{\mathrm{c}}$ ) [5]. At various points of the loops SEM microphotographs were taken (see Fig. 1 for sample No. 4). On the ascending (initial) branch we started out from the demagnetized state obtained by applying an ac field of $65 \mathrm{kA} / \mathrm{m}$ peak (exceeding the largest coercive force) which was slowly decreased to zero. On the descending branch the field was lowered from the saturation field (about $400 \mathrm{kA} / \mathrm{m}$ ). Average values of the stripe domain period $P$ were obtained by measuring in several places of the sample. We also superimposed a slowly decreasing ac field of $\max .55 \mathrm{kA} / \mathrm{m}$ on the given dc field to eliminate or to minimize hysteresis effects in $P$. In this manner we obtained "anhysteretic" values of $P$. Figure 2 shows the results for a typical sample together with results of Kerr observations for the descending branch [6] and the theoretical curve [7].

In higher fields (especially with the additional ac field) we observed the formation of either single bubble domains or in even higher fields large islands of bubble lattices in agreement with [8]. Instead of $P$ we then use the parameter $B=\sqrt{3} a / 2$ ( $a$-nearest neighbour distance between the bubbles) in figure 2. The error (the bars in Fig. 2) were mostly caused by the irregularity of the structure.
Table I. - Parameters of four low- and two medium coercive force CoCr films. Thickness: $h$ [nm], spontaneous magnetization: $M_{\mathrm{s}}[\mathrm{kA} / \mathrm{m}]$, uniaxial anisotropy: $K_{\mathrm{u}}\left[\mathrm{kJ} / \mathrm{m}^{3}\right]$, coercivity: $H_{\mathrm{c}}[\mathrm{kA} / \mathrm{m}]$, anisotropy field: $H_{\mathrm{k}}[\mathrm{kA} / \mathrm{m}]$, anhysteretic zero $P_{0}[\mathrm{~nm}]$, wall energy: $\sigma_{\mathrm{w}}\left[\mathrm{mJ} / \mathrm{m}^{2}\right]$, reduced thickness: $\tau$. The $\mathrm{CoCr}$ (78/22 at. \%) films were deposited on $\mathrm{Si}$ substrate by RF magnetron sputtering [4].

\begin{tabular}{|c|c|c|c|c|c|c|c|c|}
\hline No. & $h$ & $M_{\mathrm{s}}$ & $K_{\mathrm{u}}$ & $H_{\mathrm{c}}$ & $H_{\mathrm{c}} / H_{\mathrm{k}}$ & $P_{0}$ & $\sigma_{\mathrm{w}}$ & $\tau$ \\
\hline 1 & 493 & 360 & 110 & 8 & 0.017 & 383 & 1.6 & 62 \\
2 & 520 & 402 & 133 & 8 & 0.015 & 390 & 2.0 & 67 \\
3 & 770 & 420 & 143 & 12 & 0.022 & 452 & 2.0 & 109 \\
4 & 1230 & 404 & 143 & 8 & 0.016 & 570 & 1.8 & 175 \\
& & & & & & & & \\
5 & 300 & 420 & 142 & 24 & 0.045 & 285 & 2.0 & 42 \\
6 & 650 & 461 & 179 & 35 & 0.052 & 410 & 2.3 & 94 \\
\hline
\end{tabular}
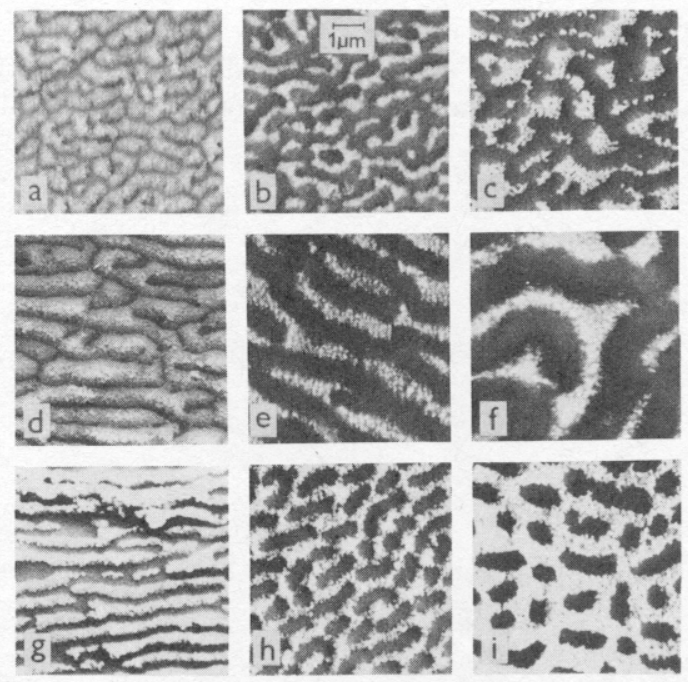

Fig. 1. - The domain structures of film No 4: ascending fields: a-c; descending fields: d-f; anhysteretic: $\mathrm{g}$-i.

The general tendency of the experimental points in figure 2 is in qualitative agreement with the theory [7]. Without the superimposed ac field the points on the 


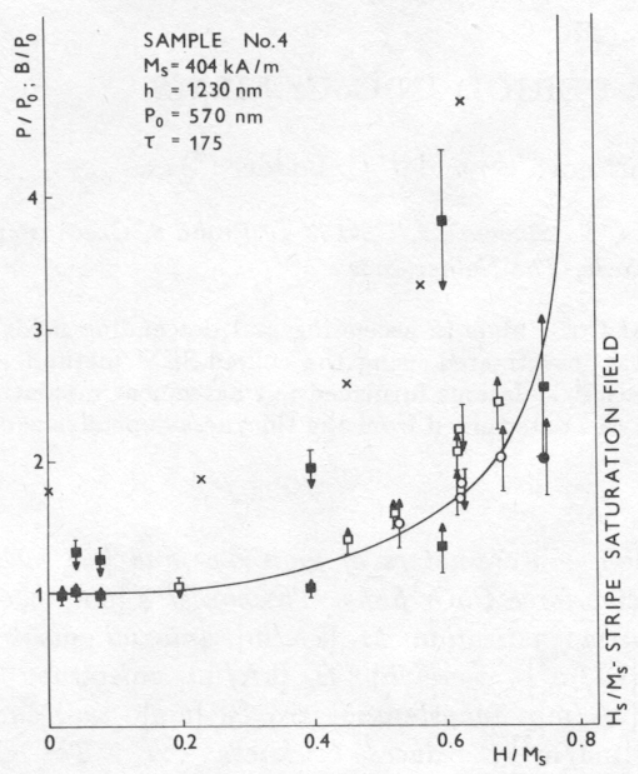

Fig. 2. - Normalized domain period $P / P_{0}$ (stripes) and $B / P_{0}$ (bubbles) vs. normalized field $H / M_{\mathrm{s}}$ (ascending $\uparrow$, descending - $\downarrow$ ). Stripes in dc field - ( $)$ ); with superim-

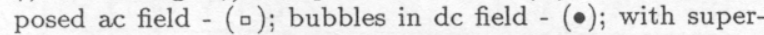
imposed ac field - (०); digitalized Kerr effect in decreasing field - $x$; full curve theory [7].

descending branch are far above-while those on the ascending branch are below the theoretical curve. This is obviously caused by the hysteresis of the film material. With the superimposed ac field fair agreement is obtained. Similar results were obtained for the other low coercivity samples while for samples No. 5, 6 larger discrepancies were found [4].

Figure 3 shows the thickness dependence of the square of the reduced period $P_{0}$ for all the samples

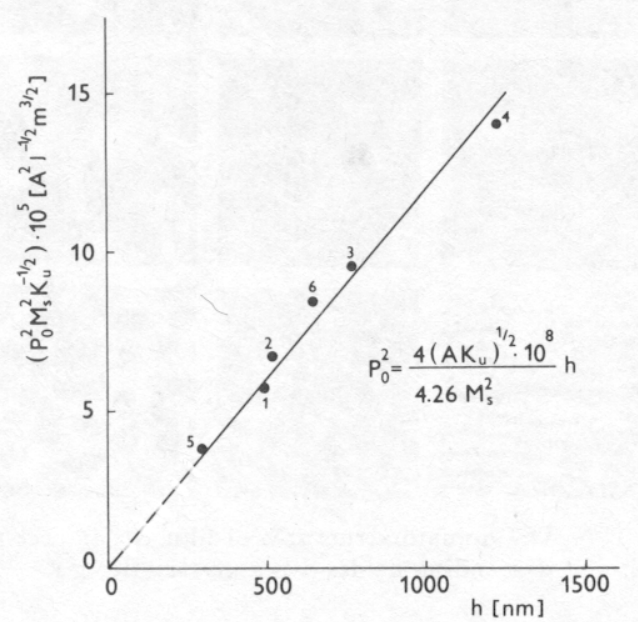

Fig. 3. - The square of the reduced stripe domain period in zero field vs. the thickness $h$ for six samples given in table I. given in table I. According to $[9,10]$ this dependence for $20<\tau<500$ follows a $1 / 2$-power law. Here $\tau$ is the reduced thickness determined from the anhysteretic zero field period: $\tau=4 \mu_{0} M_{\mathrm{s}}^{2} h / \pi \sigma_{\mathrm{W}}=37.5\left(h / P_{0}\right)^{2}$. Since all our samples are within this interval the plot of the square of the reduced period versus the thickness $h$ will be a straight line as shown in figure 3 . From [10] it also follows that no spike domains of reverse magnetization are present in our films since otherwise a 2/3-power law would hold. From the slope of the line in figure 3 we calculated the mean value of the exchange constant $A=1.64 \mathrm{pJ} / \mathrm{m}$ (including the $\mu$ correction [7]: $A=1.27 \mathrm{pJ} / \mathrm{m}$ ). The value of $A$ in [5] determined from the slope of the magnetizing curve is much larger than ours.

It is surprising that the thickness dependence of the domain period $P_{0}$ (in Fig. 3 ) is in such excellent agreement for all six samples of differing magnetic parameters, especially the coercive force and the respective domain structure.

We can conclude that:

1) the anhysteretic values of the domain period of low coercivity films are in fair agreement with the theory of Kooy and Enz;

2) the results obtained by means of the colloid-SEM method for the descending branch agree with those obtained by the Kerr technique;

3) for low and medium coercivity samples the thickness dependence of the domain period in zoro field follows the $1 / 2$-power law from which the value of the exchange constant $A$ was determined.

[1] Schmidt, F. and Hubert, A., J. Magn. Magn. Mater. 61 (1986) 307.

[2] Craik, D. J. and Griffiths, P. M., Br. J. Appl. Phys. 9 (1958) 276; Proc. Phys. Soc. 73 (1959) 1.

[3] Goto, K. and Sakurai, T., Appl. Phys. Lett. 30 (1977) 355.

[4] Simsová, J., Lodder, J. C., Kaczér, J., Gemperle, R., Jurek, K. and Tomás, I., J. Magn. Magn. Mater. 73 (1988) 131.

[5] Lodder, J. C., Wind, D., Popma, Th. J. A. and Hubert, A., IEEE Trans. Magn. MAG-23 (1987) 2055.

[6] Lodder, J. C., to be published in more details.

[7] Kooy, C. and Enz, U., Philips Res. Repts 15 (1960) 7.

[8] Cape, J. A. and Lehman, G. W., J. Appl. Phys. 42 (1971) 5732.

[9] Málek, Z. and Kamberský, V., Czech. J. Phys. 21 (1958) 416.

[10] Kaczér, J., Sov. Phys., JETP 19 (1964) 1204. 